\title{
High frequency capacitively coupled RF plasma discharge effects on the order/disorder structure of PAN-based carbon fiber
}

\author{
Ümmugül E. Güngör · Sinan Bilikmen • \\ Demiral Akbar
}

Received: 14 June 2013/Accepted: 18 December 2013/Published online: 14 May 2014

(C) The Author(s) 2014. This article is published with open access at Springerlink.com

\begin{abstract}
High-resolution confocal Raman microscopy was used to investigate the effects of nitrogen plasma on unsized high strength (HS) PAN-based carbon fiber surfaces. The fibers were treated by a high frequency $(40.68$ $\mathrm{MHz}$ ) capacitively coupled single RF-PECVD reactor under different processing conditions (exposure times, RF powers and gas pressures). It was found that the order/ disorder structure of the treated carbon fiber changed with different processing conditions. At low pressures, the degree of disordered structure increased with HF-RF power and process time. However, at high pressures, highorder structure $(I G / I T=84.51 \%)$ was observed and almost no observable structural effects appeared at long treatment time. Also, the first-order Raman-band peaks (D and $\mathrm{G}$ ) of the treated carbon fibers shifted. And, FWHM $(w D / w G)$, intensity $(I D / I G)$ and D-band relative integrated intensity $(I D / I T)$ ratios increased with ordering whereas they decreased with disordering.
\end{abstract}

Author's contributions: All Authors, UG, SB and DA, carried out the experiments and characterizations, analyzed the data, and conceptualized the research. All authors read and approved the final manuscript.

Ü. E. Güngör · S. Bilikmen

Department of Physics, Middle East Technical University, 06800 Ankara, Turkey

e-mail: ummugul@metu.edu.tr

S. Bilikmen

e-mail: bilikmen@metu.edu.tr

D. Akbar $(\bowtie)$

Advance Technology Research Center, Hacettepe University, Beytepe Campus, Beytepe, 06800 Ankara, Turkey

e-mail: dakbar@hacettepe.edu.tr

\section{Introduction}

In recent years, radio frequency plasma material processing has played an indispensable role in industrial areas [1]. Some of the applications in semiconductor manufacturing are etching (removing material from the surface), surface modification (making changes on the surface), cleaning (removing all possible undesirable residues), ashing (removing organic fragments from inorganic surfaces), and surface hardening (hardening the surface layer, without affecting the bulk properties of the material) [2]. In general, there are various methods of characterization and surface modification of the materials which are: mechanical, chemical, combustion and plasma treatment. Because the carbon fiber has only a few micrometers in diameter, it is difficult to use mechanical and combustion techniques as treatment methods. At the same time, since the popularity of chemical treatment is declining these days due to environmental pollution problems. As a result, it must be mentioned that the most convenient technique which can be used for surface modification of the carbon fibers is "plasma treatment" [3]. Furthermore, in addition to the environmental and economical benefits of the plasma glow discharges, it can maintain the properties of the mechanical bulk of carbon fiber.

In the previous literatures, the effect of the exposure time of the single RF plasma $(13.56 \mathrm{MHz})$ discharge at constant gas pressure and RF power to the crystallite structure was investigated, and it was observed that the structure of carbon fibers was not changed under plasma discharge treatment [4-7]. However, by increasing the pressure and frequency of the source, the significant structural effects of plasma treatment can be seen [3].

Carbon fiber is an artificial material containing at least $90 \%$ carbon by weight [8]. It is possible to obtain many 
different carbon fibers using different polymeric precursor materials during manufacturing; for example, cellulose, rayon, polyvinyl chloride, pitch, and polyacrylonitrile (PAN) with different mechanical properties [9]. The pitchbased fibers have high modulus with a more-ordered (graphitic) crystalline structure, whereas PAN-based fibers have high strength with a more-disordered (non-graphitic, i.e., turbostratic) structure [8-11]. Thus, Raman spectroscopy is devoted as one of the very useful technique to understand the characteristics and the properties of untreated and treated carbon fibers. Raman (vibrational molecular) spectroscopy is one of the most important analytical techniques [2], whose workout mechanism depends on the laser light scattered inelastically from the surface of a substrate. The laser spectrum carries information about the modes (especially vibrational and rotational) of molecular interaction in the substrate [12, 13]. In previous studies, many Raman-band peaks were observed in the carbon fiber structure depending on the level of graphitization, laser power, laser excitation wavelength and precursor type $[12,14,15]$. But, there are two well-known Raman bands that mostly appeared in the range of 1,200$1,700 \mathrm{~cm}^{-1}$ spectrum, which are $\mathrm{D}$ (disordered) and $\mathrm{G}$ (ordered graphitic) bands [14]. The exact peak position of these two bands is: D-band at $1,330 \mathrm{~cm}^{-1}$ and G-band at $1,585 \mathrm{~cm}^{-1}$ [12]. The reason behind the existence of the $G$ peak is the bond stretching of all pairs of $\mathrm{sp}^{2}$ atoms in both rings and chains (symmetric $E_{2} G$ vibration mode), while the $\mathrm{D}$ peak appears as a result of the breathing modes of $\mathrm{sp}^{2}$ atoms in rings (symmetric $A_{1} G$ vibration mode) [12, 14, 16]. And, the peak profile of these Raman lines is closely related to the structural properties of the analyzed carbon fiber. For example, the width of the G-band line is due to the quantity of structural disorder [17], and changes in the peak locations are observed as a result of the applied strain [18]. In addition, the relative intensity ratio of the peaks (ID/IT and $I G / I T)$ is essential to represent the amount of disorder in the carbon structure and crystallinity of carbon fibers $[14,17,20]$. The main objective of this study is to observe the structural effects of high frequency (40.68 MHz) capacitively coupled single RF nitrogen discharge on the unsized HS PAN-based carbon fiber surfaces using the Raman spectroscopy analyzing technique. It must be mentioned here that the nitrogen plasma treatments have not introduced new nitrogen functionality onto surfaces; but instead, have cleaned fiber surfaces of contaminants and successfully introducing $C / N$ functionality into fiber surfaces. The functionalization occurs within the radio frequency $(\mathrm{RF})$ plasma discharge by breaking the bonds of the fiber, and by creating reactive sites on the fiber chain which they are ready to react with active plasma species. The rapid integration of nitrogen to the surface causes formation of groups such as $\mathrm{C}=\mathrm{N}, \mathrm{N}-\mathrm{C}-\mathrm{N}$, and amine groups in plasma [21, 22].

\section{Experimental Setup}

Plasma processing set-up as shown in Fig. 1 is basically composed of a stainless steel cylindrical chamber with the dimensions $500 \times 400 \mathrm{~mm}$, two-isolated $(200 \mathrm{~mm}$ in diameter) identical aluminum electrodes, an automatic matching network, vacuum systems (rotary and Turbo pump, and high vacuum valves), a high band pass filter and RF generator. The distance between electrodes was fixed at $40 \mathrm{~mm}$. High purity $(99.995 \%)$ nitrogen gas was used as an activation gas.
Fig. 1 The diagram of a high frequency capacitively coupled single RF-PECVD reactor

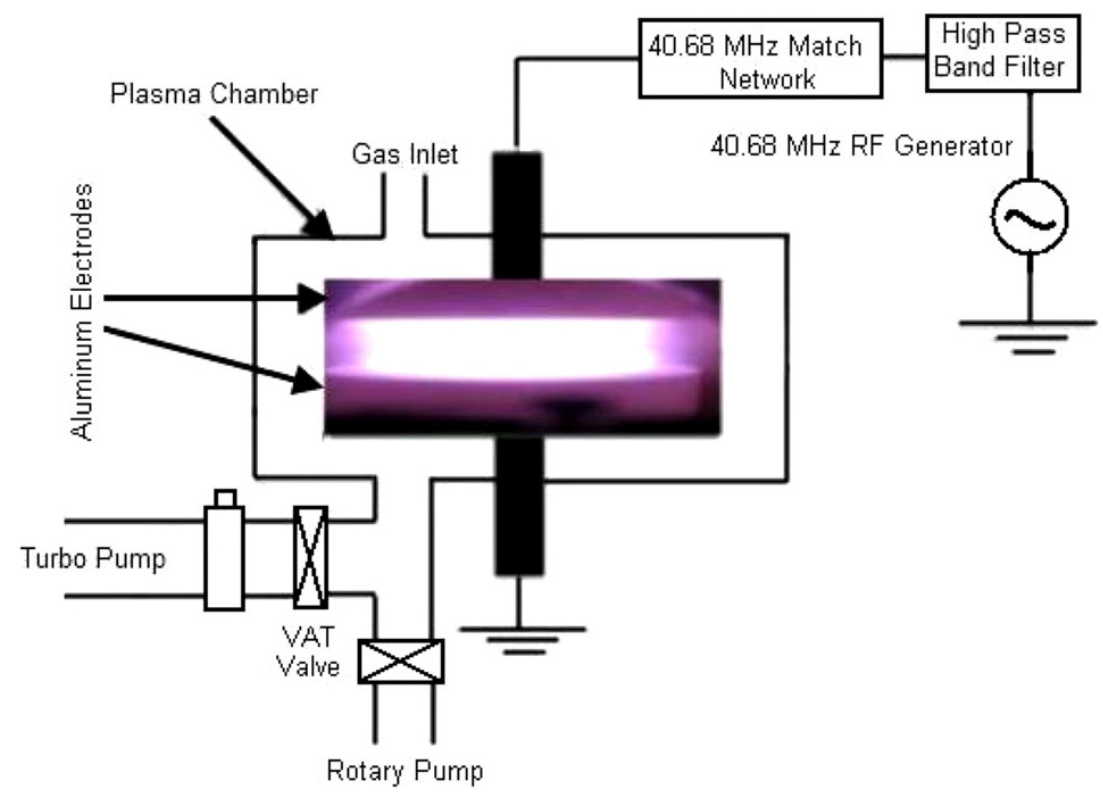


Fig. 2 Lorentzian multiplepeak-fitted Raman spectra of the treated fiber under treatment conditions 1 Torr, $200 \mathrm{~W}$ and $90 \min$

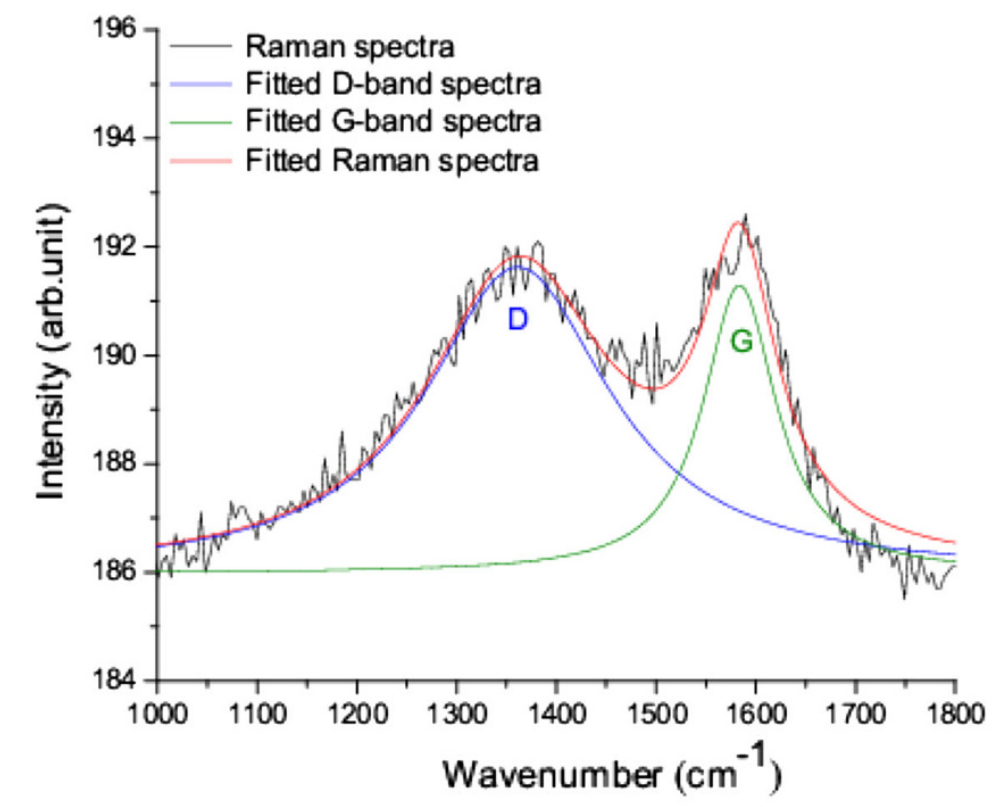

Parameters

Peaks

\begin{tabular}{lcllll}
\hline Area & Center & Width & Height & \\
\cline { 1 - 3 } 1 & 1994.6011358473 & 1361.3560585647 & 224.78913251981 & 7.0797971121318 \\
2 & 741.26485535123 & 1583.7558688153 & 88.712413833796 & 6.6669788137948
\end{tabular}

In the experimental design, a high frequency (40.68 MHz) RF source was connected to the top electrode with the automatic matching box and a high band pass filter whereas the bottom electrode was connected to the ground, as shown in Fig. 1. During plasma treatment, a non-conducting substrate (glass) with a $1-\mathrm{cm}$ thickness was used as a sample holder on the lower electrode. In this study, different operational conditions were used for the treatment of the unsized carbon fiber: The HF-RF power varied between 50 and $200 \mathrm{~W}$, the gas pressure between 0.3 and 1.0 Torr, and exposure time from 30 to $90 \mathrm{~min}$. After the plasma treatment, the confocal Raman microscopy mode (alpha300 R) of a WITEC alpha300S scanning near-field optical microscopy (SNOM) instrument was used to characterize the surface structure of the untreated (as-received) and treated carbon fiber. This mode integrates an ultrahighthroughput confocal microscope with an extremely sensitive spectroscopy system for unrivaled resolution and chemical sensitivity. It supplies nondestructive Raman imaging with optical resolution down to $200 \mathrm{~nm}$ laterally, $500 \mathrm{~nm}$ vertically and spectral resolution down to 0.02 wavenumbers. $532 \mathrm{~nm} \mathrm{He}-\mathrm{Ne}$ laser with a power $100 \mathrm{~mW}$ has been used to excite all samples. Also, $25 \times$, $50 \times$ and $100 \times$ Nikon objective lenses have been used to focus the laser beam onto the fiber surface. The laser light excites the surface by inducing vibrations of the chemical bonds within the samples. And, the spectrometer collects scattered lights from the surface after filtering, and sends them to the CCD camera detector connected to a powerful computer and a WITec special software system. The scanned Raman spectra started from 0 to $3,400 \mathrm{~cm}^{-1}$ to detect the first-order D and $G$ bands of the carbon fibers. The Origin Pro8 software program was used for data analyzing according to the Lorentz curve fitting $[3,18]$ model that is defined as: 

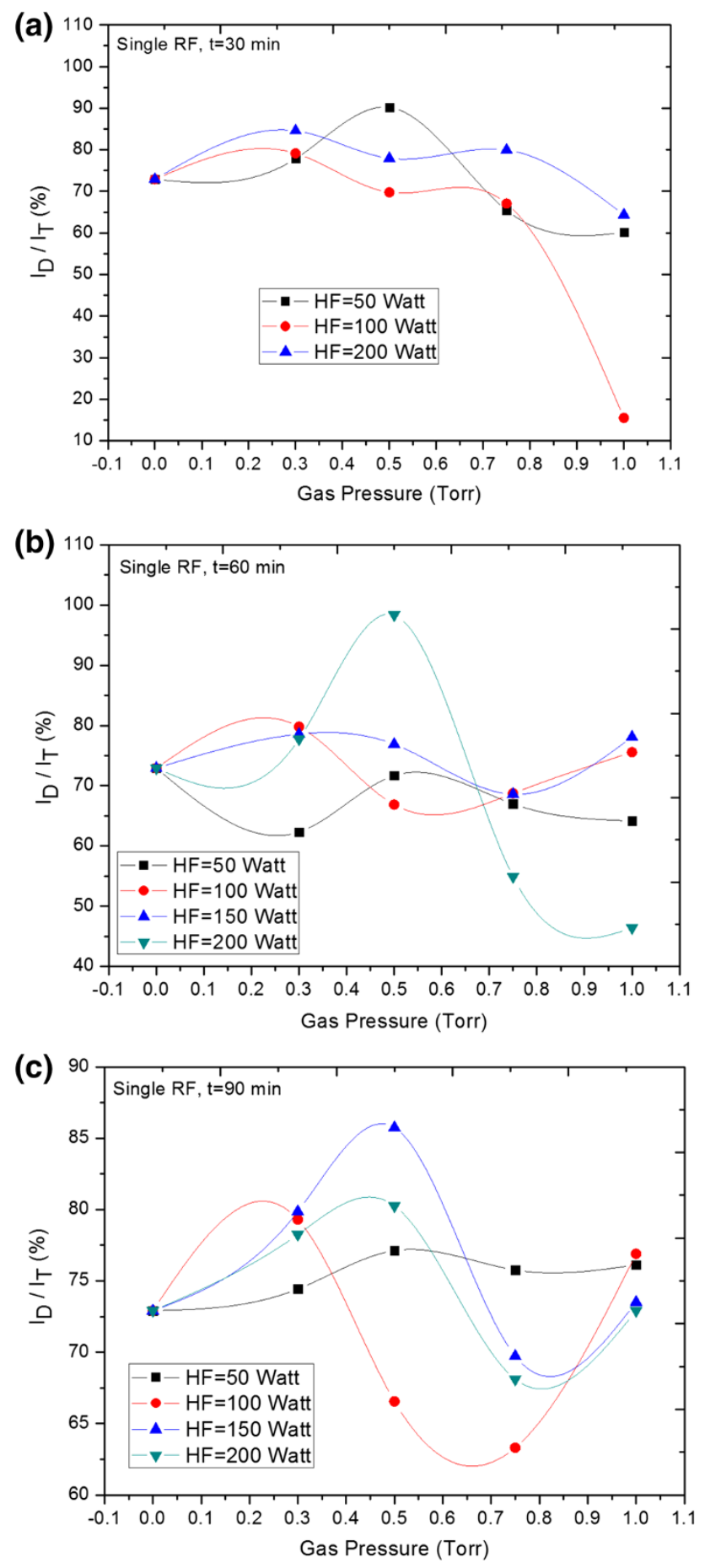

Fig. 3 Effects of gas pressure and HF-RF power on the order/ disorder structure of the carbon fibers treated under different exposure times; a $30 \mathrm{~min}$, b $60 \mathrm{~min}$ and c $90 \mathrm{~min}$

$y=y_{0}+\frac{2 A}{\pi} \frac{\omega}{4\left(x-x_{0}\right)^{2}+\omega^{2}}$

where $y_{0}$ is the baseline offset, $A$ is the total area under the curve from the baseline, $x_{0}$ is the center of the peak and $\omega$
Table 1 RF plasma processing treatment conditions of unsized PANbased carbon fibers samples (a detail calculation is given in Table 2)

\begin{tabular}{lrll}
\hline Sample & Power $(\mathrm{W})$ & Pressure (Torr) & Time (min) \\
\hline Disorder & & & \\
1 & 200 & 0.5 & 60 \\
2 & 150 & 0.75 & 30 \\
3 & 50 & 0.5 & 30 \\
4 & 150 & 0.5 & 90 \\
5 & 200 & 0.3 & 30 \\
6 & 150 & 0.3 & 30 \\
7 & 200 & 0.5 & 90 \\
Order & & & \\
8 & 100 & 1 & 30 \\
9 & 200 & 1 & 60 \\
10 & 200 & 0.75 & 60 \\
11 & 50 & 0.3 & 60 \\
12 & 100 & 0.75 & 90 \\
13 & 50 & 1 & 60 \\
14 & 200 & 1 & 30 \\
\hline
\end{tabular}

is the full width of the peak at half height (FWHM), since this fitting is usually preferred in the analysis of disordered carbon crystal [19]. Among these fitted parameters, the area $(A)$ represents the integrated intensity of the peaks which can be used in the calculation of relative integrated intensity (curve area) ratios: $I D / I T$ and $I G / I T$, where $I D$ is the integrated intensity of the D-peak, $I G$ is the integrated intensity of the G-peak and $I T$ is total the integrated intensity of these two peaks $(I D+I G)$. An example of fitted Raman spectra with its parameters is shown in Fig. 2.

\section{Results and discussion}

In this study, the effects of gas pressure, RF power and exposure time on the order/disorder crystalline structure of treated HS PAN-based carbon fibers were investigated. Furthermore, improvement of the interfacial properties of the treated fibers was examined by analyzing peak positions, widths and curve area ratios of the first-order Raman bands.

Crystalline structure

\section{Effects of gas pressure and $R F$ power}

In short treatment time The degree of the disordered structure increases at low pressure, as in Fig. 3a, but, generally there is no observable effect of HF-RF power. 

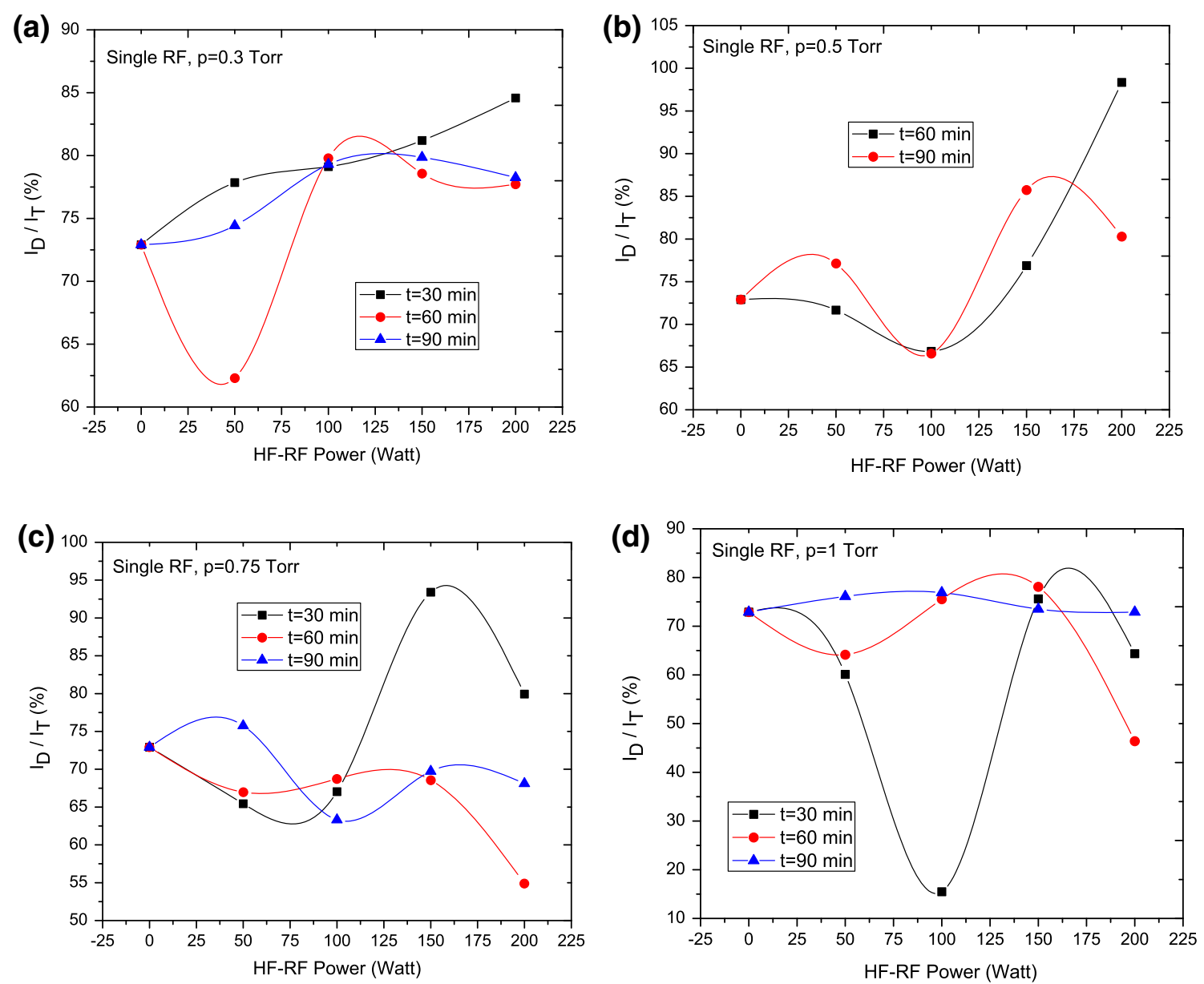

Fig. 4 Effects of HF-RF power and exposure time on the order/disorder structure of the carbon fibers treated under different gas pressures: a 0.3 Torr, b 0.5 Torr, $\mathbf{c} 0.75$ Torr and $\mathbf{d} 1$ Torr

However, at high pressure, the degree of ordered structure increases and the effect of power is seen by increasing pressure. Thus, the highest ordered structure $(84.51 \%)$ is obtained under the treatment condition 1 Torr and $100 \mathrm{~W}$.

In long treatment time Figure $3 \mathrm{~b}$, $\mathrm{c}$ shows that the variations in the disordering and the effect of HF-RF power are almost the same as that of short treatment at low pressure. At high pressure, the effect of HF-RF power is clearly seen with pressure which disappears at long treatment time $(90 \mathrm{~min})$ at 1 Torr. The ordered degree of the treated fiber increases with increasing pressure except at $100 \mathrm{~W}$. At $60 \mathrm{~min}$ and $200 \mathrm{~W}$, the highest ordered $(I G / I T=53.61 \%)$ structure was seen under treatment conditions 1 Torr. Meanwhile, the highest disordered structure $(I D / I T=98.35 \%$, as in Table 1$)$ is obtained around 0.5 Torr. Generally, the degree of disordering increases with increasing the gas pressure (for pressure range $<0.3$ Torr) due to collisionless (nonOhmic) electron heating whereas the degree of ordering increases with increasing pressure ( $>0.3$ Torr) because of collisional (Ohmic) electron heating [20]. Because the transition point of the plasma from collisionless (alpha mode) collisional (gamma mode) which causes an increase of the number of energetic particles and nitrogen enrichment.

\section{Effects of RF power and exposure time}

At low gas pressure The degree of disordered structure increases with HF-RF power, as in Fig. 4a, b. Since HFRF power increases the excitation and rotational temperatures of the neutral nitrogen gas which causes an increase in the density of the vibrational excited particles [23, 24]. These excited species destroy the basal plane of the fiber leading to an increase in the number of carbon atoms in the edge plane of the fiber that occurs in a high-level disordered structure $[18,25,26]$. In addition, a different molecular rearrangement can be observed in the carbon fiber structure with increasing the RF power and treatment time: carbon to nitrogen bonds have triple bonds $(\mathrm{C} \equiv \mathrm{N})$ at 

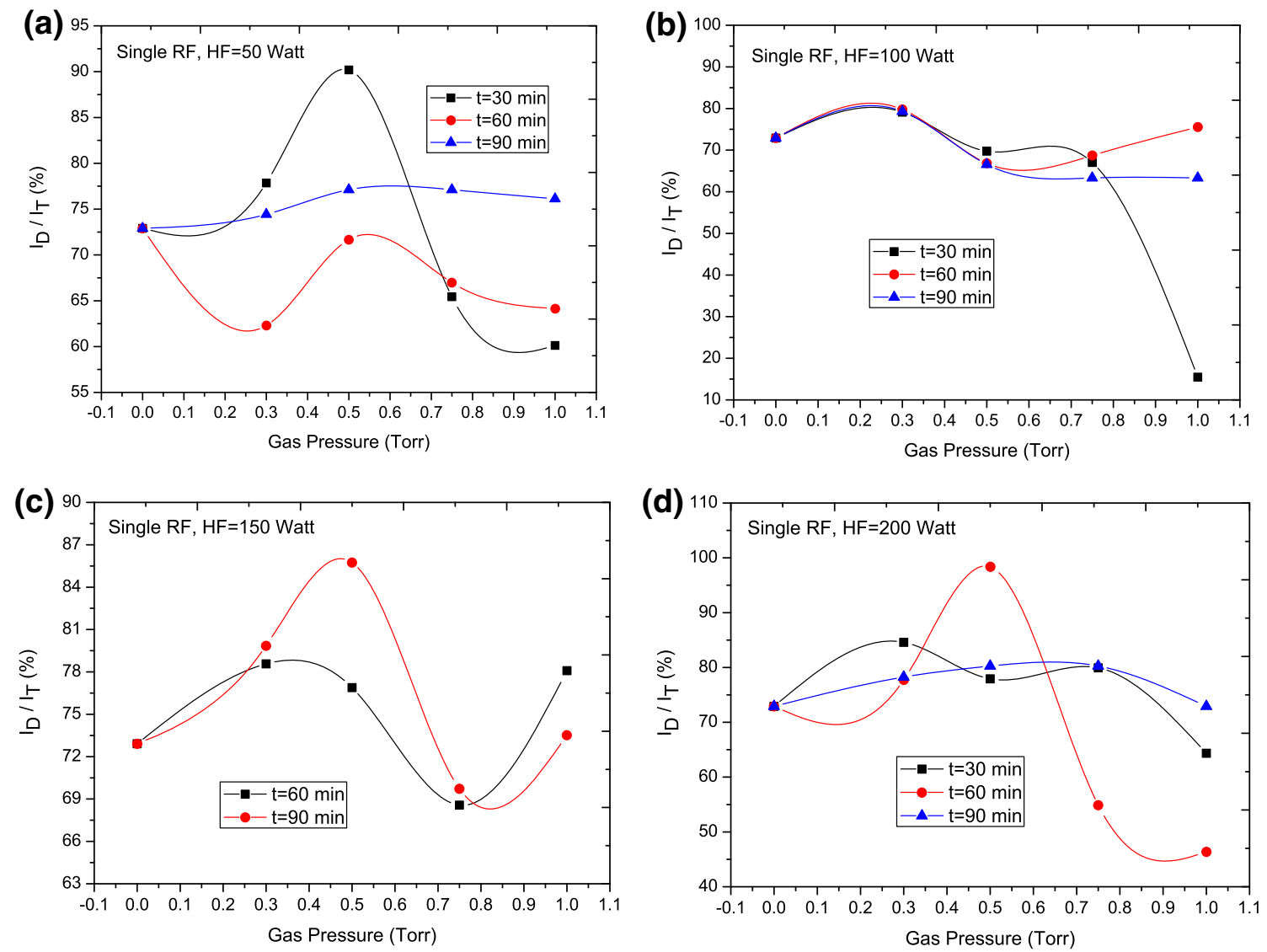

Fig. 5 Effects of gas pressure and exposure time on the order/disorder structure of the carbon fibers treated under different HF-RF powers: a $50 \mathrm{~W}$, b $100 \mathrm{~W}$, c $150 \mathrm{~W}$ and d $200 \mathrm{~W}$

Table 2 Lorentz-fitted parameters of D and G Raman bands of untreated (as-received) and treated fibers listed in Table 2:

\begin{tabular}{|c|c|c|c|c|c|c|c|c|}
\hline Carbon fiber & $\begin{array}{l}x_{o}-D \\
\left(\mathrm{~cm}^{-1}\right)\end{array}$ & $\begin{array}{l}x_{o}-G \\
\left(\mathrm{~cm}^{-1}\right)\end{array}$ & $\begin{array}{l}\omega_{D} \\
\left(\mathrm{~cm}^{-1}\right)\end{array}$ & $\begin{array}{l}\omega_{G} \\
\left(\mathrm{~cm}^{-1}\right)\end{array}$ & $\omega_{D} / \omega_{G}$ & $I D / I G$ & $\begin{array}{l}I D / I T \\
(\%)\end{array}$ & $\begin{array}{l}I D / I T \\
(\%)\end{array}$ \\
\hline Untreated & 1,362 & 1,584 & 225 & 89 & 2.53 & 2.69 & 72.91 & 27.09 \\
\hline \multicolumn{9}{|l|}{ Disorder } \\
\hline Sample 1 & 1,437 & 1,560 & 926 & 68 & 13.72 & 59.46 & 98.35 & 1.65 \\
\hline Sample 2 & 1,388 & 1,566 & 603 & 96 & 6.27 & 14.17 & 93.41 & 6.59 \\
\hline Sample 3 & 1,367 & 1,562 & 527 & 97 & 5.44 & 9.19 & 90.19 & 9.81 \\
\hline Sample 4 & 1,355 & 1,560 & 384 & 96 & 4.01 & 6.01 & 85.74 & 14.26 \\
\hline Sample 5 & 1,350 & 1,572 & 335 & 77 & 4.35 & 5.49 & 84.58 & 15.42 \\
\hline Sample 6 & 1,365 & 1,589 & 293 & 84 & 3.50 & 4.32 & 81.20 & 18.80 \\
\hline Sample 7 & 1,349 & 1,561 & 298 & 103 & 2.89 & 4.07 & 80.26 & 19.74 \\
\hline \multicolumn{9}{|l|}{ Order } \\
\hline Sample 8 & 1,351 & 1,560 & 4 & 16 & 0.25 & 0.18 & 15.49 & 84.51 \\
\hline Sample 9 & 1,353 & 1,560 & 41 & 33 & 1.26 & 0.87 & 46.39 & 53.61 \\
\hline Sample 10 & 1,359 & 1,554 & 92 & 64 & 1.42 & 1.22 & 54.88 & 45.12 \\
\hline Sample 11 & 1,354 & 1,562 & 160 & 86 & 1.86 & 1.65 & 62.30 & 37.70 \\
\hline Sample 12 & 1,350 & 1,554 & 151 & 86 & 1.76 & 1.73 & 63.31 & 36.69 \\
\hline Sample 13 & 1,355 & 1,558 & 161 & 89 & 1.82 & 1.79 & 64.15 & 35.85 \\
\hline Sample 14 & 1,353 & 1,560 & 168 & 87 & 1.93 & 1.81 & 64.36 & 35.64 \\
\hline
\end{tabular}



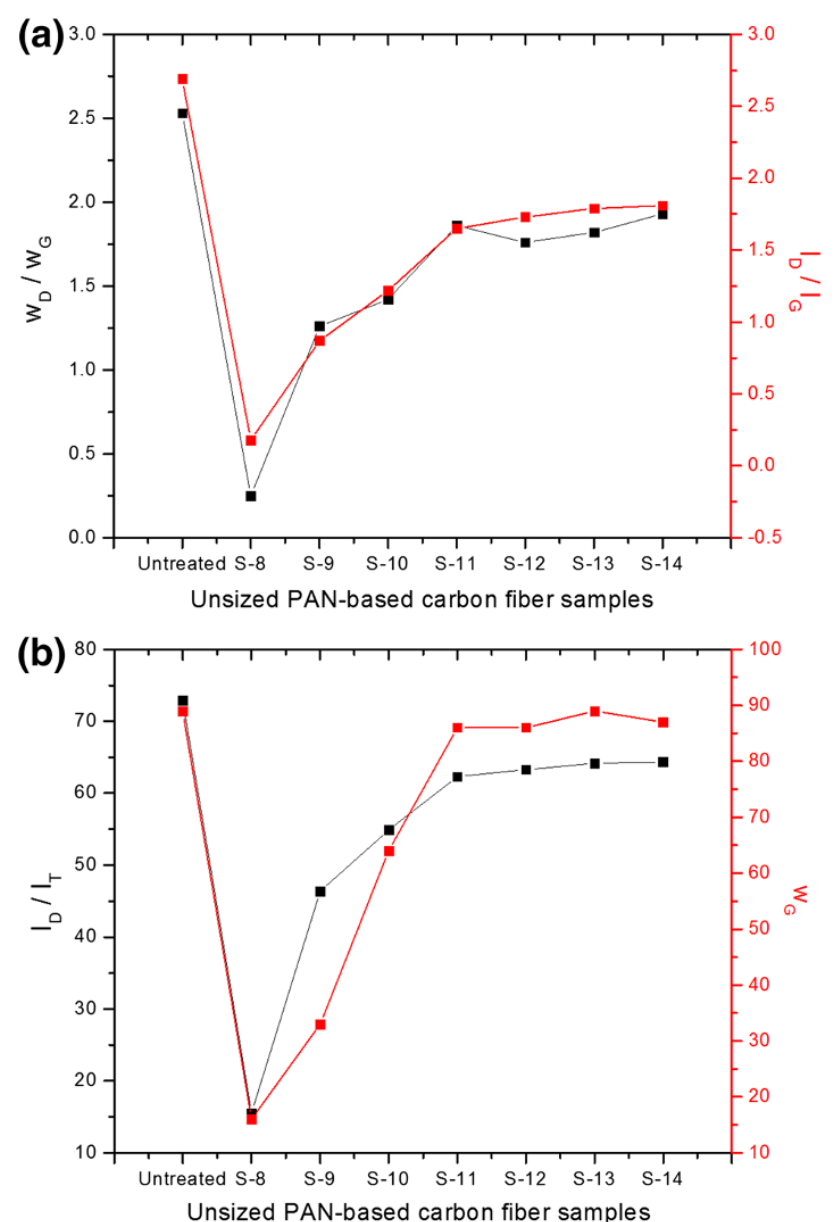

Fig. 6 a FWHM $(w D / w G)$ and the integrated intensity $(I D / I G)$ ratios, and $\mathbf{b}$ the relative integrated intensity $(I D / I T)$ ratio and FWHM (G) of the high-ordered carbon fibers listed in Table 1. ('Sample' is abbreviated as "S")

30 min, but it can be changed to double bond $(\mathrm{C}=\mathrm{N})$ by increasing the exposure time. This result will be investigated in more detail in the future.

At high gas pressure From Fig. 4c, d, the degree of the ordered structure increases with HF-RF power only during short time treatment $(30 \mathrm{~min})$. But, there was almost no power effect on the structure during the ninety-minute treatment at 0.75 and 1 Torr.

\section{Effects of gas pressure and exposure time}

At low $H F-R F$ power From Fig. 5a, b, at low HF-RF power $(50 \mathrm{~W})$ and with a short time treatment, the degree of disordering increases with low pressure whereas it decreases by increasing the pressure. Moreover, with a long-time treatment, the degree of ordering increases by increasing pressure. At HF-RF power $(100 \mathrm{~W})$, the treatment time effect is clearly seen with high gas pressure especially with a short process time. With a 90 -min process time, there is no pressure influence on the crystalline structure.

At high HF-RF power As shown in Fig. 5c, d, the level of disordering increases by decreasing the gas pressure. And, the crystalline structure of the carbon fibers treated for $60 \mathrm{~min}$ becomes more ordered with high pressure. Furthermore, the pressure effect of 90-min treatment time on the disordering structure decreases by increasing the high HF-RF power.

\section{Interfacial properties}

Since the obtained results are very complicated and require more detailed work, only samples which show an increment higher than $8 \%$ of order/disorder crystalline structure have been selected at this stage of our work, as in Table 1, and fitted Raman-band parameters of these fibers are shown in Table 2.

According to previous Raman spectral analysis, central peak positions $\left(x_{o}\right)$ of both Raman bands were shifted due to enharmonic distribution, decrease in bond modulus and increase in stress $[12,27,28]$ and also increase in crystallite size. Moreover, FWHM (G) of the treated fibers decrease due to an increase in fiber modulus $[8,16,17]$ and having more-ordered molecular structure. According to the low value of the FWHM $(w D / w G)$ and the integrated intensity $(I D / I G)$ ratios, it can be inferred that the tensile strain of the treated carbon fibers increase [10, 12, 16, 29]. Furthermore, the percentage increase in the relative integrated intensity ratio of G-band (or decrease in $I D / I T$ ) shows to a higher degree of graphitization, fiber modulus and crystallite size $[4,18,29-31]$. And, a similar relation between these Raman parameters can also be seen in Fig. 6 . Therefore, it can obviously be inferred that tensile strain, fiber modulus and crystallite size of the high-ordered carbon fibers listed in Table 1 increase with nitrogen highfrequency RF plasma treatment under different processing conditions. Also, sample 8 (in Table 1) has the highest property and ordered structure due to crystallization [9, 17]. According to these results, it is very important to mention that there will be subsequent extensive measurement and analysis in future work.

\section{Conclusion}

In conclusion, this study points out that high-frequency capacitively coupled RF nitrogen plasma treatment processes have unexpected effects on structural ordering/disordering and some interfacial properties of the unsized HS PAN-based carbon fiber. Different structural changes were observed with different gas pressures, RF powers, and exposure times that are: (a) In short time treatment, the 
amount of disordered structure increases with increasing HF-RF power at low pressure due to the increase in excitation and rotational temperatures of the neutral nitrogen molecular gas and also non-Ohmic electron heating. (b) In long time treatment, the degree of ordered structure increased with HF-RF power with high pressure due to Ohmic electron heating. (c) 90-min treatment is too long to observe structural changes at very high pressure, and interfacial properties of the carbon fibers were improved more with high pressure and high power.

Acknowledgments We would like to thank Prof. Dr. Ural Akbulut and Prof. Dr. Erdal Bayramlı for their support in this work and also Bilkent National Nanotechnology Research Center (UNAM) for allowing the use their confocal Raman microscopy device.

Open Access This article is distributed under the terms of the Creative Commons Attribution License which permits any use, distribution, and reproduction in any medium, provided the original author(s) and the source are credited.

\section{References}

1. Lieberman, M.A., Lichtenberg, A.J.: Principles of plasma discharges and materials processing. Wiley, New York (1994)

2. Montes-Moran, M.A., Martinez-Alonso, A., Tascon, J.M.D., Young, R.J.: Effects of plasma oxidation on the surface and interfacial properties of ultra-high modulus carbon fibers. Comp. Part A: Appl. Sci. Manuf. 15, 361-371 (2001)

3. Li, R., Ye, L., Mai, Y.W.: Application of plasma technologies in fibre-reinforced polymer composites: a review of recent developments. Comp. Part A: Appl. Sci. Manuf. 28(1), 73-86 (1997)

4. Sarraf, H., Louda, P., Skarpova, L.: Surface modification of carbon fiber. J. Ach. Mater. Manuf. Eng. 25, 24-30 (2007)

5. Nohara, L.B., Filho, G.P., Nohara, E.L., Kleinke, M.U., Rezende, M.C.: Evaluation of carbon fiber surface treated by chemical and cold plasma processes. Mater. Res. 8, 281-286 (2005)

6. Jang, J., Yang, H.: The effect of surface treatment on the performance improvement of carbon fiber/polybenzoxazine composites. J. Mater. Sci. 35, 2297-2303 (2000)

7. Chang, T.C.: Plasma surface treatment in composites manufacturing. J. Ind. Tech. 15, 1-7 (1999)

8. Minus, M.L., Kumar, S.: The processing, properties and structure of carbon fibers. J. Mater. Sci. 57(2), 52-58 (2005)

9. Huang, X.: Fabrication and properties of carbon fibers. Mater. Rev. 2, 2369-2403 (2009)

10. Chen, J.C., Harrison, I.R.: Modification of polyacrylonitrile (PAN)carbon fiber precursor via post-spinning plasticization and stretching in dimethyl formamide (DMF). Carbon 40, 25-45 (2002)

11. Oya, N., Johnson, D.J.: Longitudinal compressive behavior and microstructure of PAN-based carbon fibers. Carbon 39, 635-645 (2001)

12. Blum, Jr F.D.: Condition assessment of carbon fiber composites using Raman spectroscopy. Columbia, University of Missouri-Ms Thesis (2009)
13. Washer, G., Blum Jr, F.: Raman spectroscopy for the nondestructive testing of carbon fiber. Res. Lett. Mater. Sci. 2008, 1-3 (2008)

14. Matthews, M.J., Pimenta, M.A., Dresselhaus, G., Dresselhaus, M.S., Endo, M.: Origin of dispersive effects of the Raman D band in carbon materials. Phys. Rev. B 59, R6585-R6588 (1999)

15. Galiotis, C., Batchelder, D.N.: Strain dependence of the first order and second order Raman spectra of carbon fibers. J. Mater. Sci. Lett. 7, 545-547 (1988)

16. Ferrari, A.C.: Raman spectroscopy of graphene and graphite: disorder, electron-phonon coupling, doping and nonadiabatic effects. Elsevier Solid State Commun. 143, 47-57 (2007)

17. Melanitis, N., Tetlow, P.L., Galiotis, C.: Characterization of PAN-based carbon fibers with laser Raman spectroscopy.1. Effect of processing variables on Raman band profiles. J. Mater. Sci. 31, 851-860 (1996)

18. Thomsen, C., Reich, S.: Ab initio determination of the phonon deformation potentials of grapheme. Phys. Rev. B 65, 073403 (2002)

19. Ferrari, A.C., Robertson, J.: Interpretation of Raman spectra of disordered and amorphous carbon. Phys. Rev. B 61, 14095 (2000)

20. Kim, H.C., Lee, J.K.: Mode transition induced by low-frequency current in dual-frequency capacitive discharges. Phys. Rev. Lett. 93, 085003 (2004)

21. Smiley, R.J., Delgass, W.N.: XPS and sem characterization of pan-based carbon fibers treated in oxygen and nitric oxide plasmas. MRS Proc. 318, 361 (1993). doi:10.1557/PROC-318-361

22. Wade, G.A., Cantwell, W.J.: Adhesive bonding and wettability of plasma treated, glass fiber-reinforced nylon-6,6 composites. J. Mater. Sci. Lett. 19, 1829 (2000)

23. Huang, X.J., Xin, Y., Yang, L., Ye, C., Yuan, Q.H., Ning, Z.Y.: Analysis of optical emission spectroscopy in a dual-frequency capacitively coupled CHF3 plasma. Phys. Plas. 16, 043509-043513 (2009)

24. Huang, X.J., Xin, Y., Yang, L., Yuan, Q.H., Ning, Z.Y.: Spectroscopic study on rotational and vibrational temperature of $\mathrm{N} 2$ and $\mathrm{N} 2+$ in dual-frequency capacitively coupled plasma. Phys. Plas. 15:113504-113509 (2008)

25. Montes-Moran, M.A., Young, R.J.: Raman spectroscopy study of HM carbon fibres: effect of plasma treatment on the interfacial properties of single fiber/ epoxy composites Part I: Fibre characterization. Carbon 40, 845-855 (2002)

26. Reich, S., Jantoljak, H., Thomsen, C.: Shear strain in carbon nanotubes under hydrostatic pressure. Phys. Rev. B 61(R13), 389-392 (2000)

27. Mitra, V.K., William, M.R., Baughman, R.H.: A laser Raman study of the stress dependence of vibrational frequencies of a monocrystalline polydiacetylene. J. Chem. Phys. 66, 2731-2736 (1977)

28. Angoni, K.: A study of highly ordered carbons by use of macroscopic and microscopic Raman spectroscopy. J. Mater. Sci. 33, 3693-3698 (1998)

29. Ungar, T., Gubicza, J., Tichy, G., Pantea, C., Zerda, T.W.: Size and shape of crystallites and internal stresses in carbon blacks. Composites: Part A 36, 431-436 (2005)

30. Paris, O., Loidl, D., Peterlik, H.: Texture of PAN- and pitchbased carbon fibers. Carbon 40, 551-555 (2002)

31. Miyagawa, H., Kamiya, D., Sato, C., Ikegami, K.: Strain measurement for Raman-inactive substrates with $\mathrm{PbO}$ thin films using Raman coating method. J. Mater. Sci. 34, 105-110 (1999) 